\title{
Diagnosing fevers of unknown origin with cervical lymphadenopathy: A case report of Kikuchi-Fujimoto disease
}

\author{
Andres Gottfried-Blackmore ${ }^{1 *}$ and Vishal H. Dodia ${ }^{2}$ \\ ${ }^{1}$ Division of Gastroenterology and Hepatology, Stanford University School of Medicine, Stanford, CA, USA; ${ }^{2}$ Department of Internal Medicine, Weill Cornell New York \\ Presbyterian Hospital, New York, NY, USA
}

\begin{abstract}
Introduction: Fevers of unknown origin (FUO) are often attributed to an infectious, malignant, or autoimmune process. Kikuchi-Fujimoto disease (KFD) is a rare, self-limited, autoimmune disorder with characteristic necrotizing lymphadenitis and prolonged fevers. We present a case report of this disorder with a discussion on the role of lymph node biopsy in working-up FUO with cervical lymphadenopathy (LAD). Case Report: We report the case of a 36-year-old woman who presented with 2 months of FUO associated with tender cervical LAD, arthralgias, and myalgias. On admission, the patient underwent extensive laboratory evaluation noting leukopenia, elevated systemic inflammatory markers, and a negative infectious work-up. The diagnosis was eventually made following excisional lymph node biopsy, which confirmed KFD. Conclusion: Although a rare autoimmune disease, KFD should remain on the differential diagnosis of patients with FUO with cervical LAD. This case report exemplifies how lymph node biopsy can expedite diagnosis in patients presenting with prolonged fevers and cervical LAD after initial negative laboratory evaluation. (Hosp Med Clin Manag. 2019;12:40-6)

Corresponding author: Andres Gottfried-Blackmore, andresg@ stanford.edu
\end{abstract}

Key words: Fever of unknown origin. Lymphadenopathy. Histiocytic necrotizing lymphadenitis. Lymph node biopsy.

\section{RESUMEN}

Introducción: Las fiebres de origen desconocido a menudo se atribuyen a un proceso infeccioso, maligno o autoinmune. La enfermedad de Kikuchi-Fujimoto es un trastorno autoinmune raro, limitado, con una característica linfadenitis necrotizante y fiebres prolongadas. Presentamos un caso clínico de este trastorno con una discusión sobre el papel que juega la biopsia de los ganglios linfáticos en la investigación de fiebres de origen desconocido con linfadenopatía cervical. El caso: Una mujer de 36 años que se presentó con 2 meses de fiebres de origen desconocido con linfadenopatía cervical, artralgias y mialgias. En el momento del ingreso, el paciente se sometió a una

\section{Correspondencia:}

*Andres Gottried-Blackmore

E-mail: andresg@stanford.edu
Date of reception:25-01-2019

Date of acceptance: 13-03-2019

DOI: 10.24875/HMCM.19000183 
extensa evaluación de laboratorio que observó leucopenia, marcadores inflamatorios sistémicos elevados y un diagnóstico infeccioso negativo. El diagnóstico se hizo finalmente después de la biopsia de ganglio linfático por escisión, que confirmó la enfermedad de Kikuchi-Fujimoto. Conclusión: Aunque es una enfermedad autoinmune poco común, la enfermedad de Kikuchi-Fujimoto debe permanecer en el diagnóstico diferencial de pacientes con fiebres de origen desconocido con linfadenopatía cervical. El caso presentado ilustra cómo la biopsia de ganglios linfáticos puede acelerar el diagnóstico en pacientes que presentan fiebres prolongadas y linfadenopatía cervical después de una evaluación inicial de laboratorio negativa.

Palabras clave: Fiebre de origen desconocido. Linfadenopatía. Linfadenitis necrotizante histiocítica. Biopsia de ganglios linfáticos.

\section{INTRODUCTION}

Fevers of unknown origin (FUO), commonly defined by temperatures above $38.3^{\circ} \mathrm{C}$ lasting for at least 3 weeks with a negative initial work-up, are usually caused by infections, malignancy, or connective tissue diseases. Infectious causes include tuberculosis, abscesses, typhoid fever, and malaria. The most prevalent connective tissue diseases in FUO are adult Still's disease and giant cell arteritis in younger and older patients, respectively. Common malignancies presenting as FUO are lymphoma (especially non-Hodgkin's), leukemia, and hepatocellular $\mathrm{CA}^{1}$. Critical to diagnosis is a careful history that should include travel, animal exposure, immunosuppression, drugs/antimicrobials, localizing symptoms, a meticulous physical examination, and periodic re-evaluation of the patient. High erythrocyte sedimentation rates (ESRs), although non-specific, can suggest serious infections or inflammatory processes. Imaging and biopsy are often valuable adjuncts to diagnosis. FUO associated with cervical lymphadenopathy (LAD) narrows the differential diagnosis. Anterior cervical LAD occurs following a variety of infections of the head and neck or systemic infections such as cytomegalovirus (CMV) infection, or toxoplasmosis. Posterior cervical LAD is associated with tuberculosis, rubella, Iymphoma, Kikuchi-Fujimoto disease (KFD), or head and neck malignancies such as lymphomas or metastatic squamous cell carcinoma². Infectious mononucleosis caused by Epstein-Barr virus (EBV) infection can lead to anterior and/or posterior cervical LAD. We describe a patient who presented with 2 months of $F \cup O$, arthralgias, myalgias, and tender cervical $L A D$ that was diagnosed with KFD following an extensive inpatient work-up. Lymph node biopsy was critical to diagnosis, which allowed for patient discharge and appropriate supportive care of this rare self-limiting autoimmune disease.

\section{CASE REPORT}

This patient, a 36-year-old Bangladeshi woman who immigrated to New York City at age 27 , presented with a 2-month history of persistent fevers of up to $38^{\circ} \mathrm{C}$ associated with sore throat and painful lumps in her neck. 2 weeks into her illness, she was diagnosed at an outside hospital with tonsillitis and sent home on a 10-day course of amoxicillin, which she completed. However, her fevers and tender cervical LAD persisted. 1 month before admission, she presented to our emergency department (ED) at which time a computed tomography head/neck showed prominent posterior and anterior cervical LAD but no abscess (Fig. 1). She was discharged with analgesics and antipyretics and outpatient follow-up in the Hematology-Oncology Clinic. In the following 4 weeks, she presented 2 more times to the ED with persistent fevers, having negative blood cultures from these visits. In addition, in the 2 weeks before admission, she complained of morning joint stiffness and bilateral joint pain in the knees, hips, and hands, with intermittent diffuse myalgias. She also reported night sweats and chills associated with the fevers, which responded to acetaminophen, as well as non-localizing occasional abdominal pain and decreased appetite. There was no history of nausea, vomiting, diarrhea, weight loss, rashes, swollen/hot joints, confusion, neck stiffness, dysphagia, headaches, chest pain, dyspnea, cough, dysuria, or recent travel. Of note, her mother had come from Bangladesh to stay at her home about 2 months before admission, and her young son had been diagnosed and treated for a viral meningitis 2 weeks before admission; the patient did not receive any prophylaxis treatment. She reported no pets at home.

On the day of admission, she visited the Hematology-Oncology Clinic and was found to be febrile, 

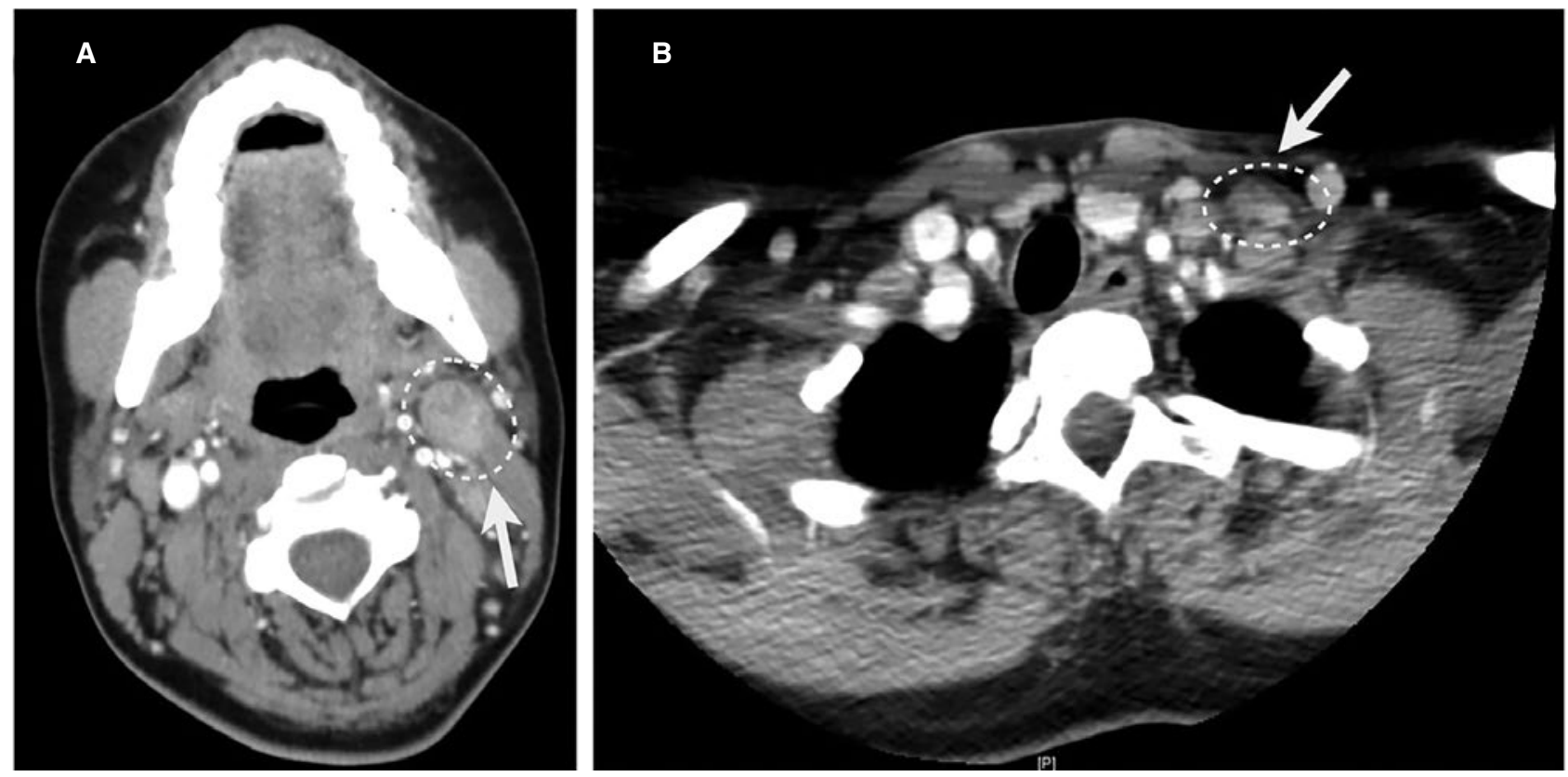

Figure 1. Head and neck computed tomogram w/ contrast. A: Transverse view of upper neck showing a $1.3 \mathrm{~cm} \times 2.0 \mathrm{~cm}$ prominent cervical lymph node. B: Transverse view of the neck base outlining one of various enlarged anterior cervical lymph nodes (circled node is $0.9 \mathrm{~cm} \times 1.2 \mathrm{~cm}$ ). No evidence of fluid collections or abscesses was detected.

ill-appearing, and with a systolic blood pressure of $94 \mathrm{mmHg}$, so she was sent to the ED for evaluation and admission. Her past medical history was significant for rheumatic fever, right elbow osteomyelitis, and a positive PPD status after a 9-month INH therapy at age 16 while living in Bangladesh. Her only medications were ibuprofen and acetaminophen, and she had no known drug allergies. Family history was non-contributory. The patient works as a hotel hostess, lives with her husband and two children, and denied tobacco, alcohol, or illicit drug use. Her physical examination was notable for fever of $38.4^{\circ} \mathrm{C}$, heart rate of $98 / \mathrm{min}$, blood pressure of $101 / 64 \mathrm{mmHg}$, tender cervical bilateral LAD with a $2 \mathrm{~cm} \times 2 \mathrm{~cm}$ exquisitely tender mass under the left mastoid, and joints tender to palpation but with no effusions. Her laboratory results showed marked leukopenia with a white blood cell of $2900 / \mathrm{mm}^{3}$, mild anemia with hemoglobin of $11.2 \mathrm{~g} / \mathrm{dL}$, elevated lactate dehydrogenase (LDH) of $496 \mathrm{U} / \mathrm{L}$, ESR of $84 \mathrm{~mm} / \mathrm{h}$, mild transaminitis with aminotransferase (AST) of $75 \mathrm{IU} / \mathrm{L}$ and alanine aminotransferase (ALT) of $56 \mathrm{IU} / \mathrm{L}$, and a protein-albumin gap of 4.2 (7.0-2.8). Electrolytes, coagulation tests, C-reactive protein levels, and urine analysis were all normal. On hospital day 1 , antipyretics were discontinued and the patient remained afebrile for the rest of the 6-day admission. Extensive infectious work-up showed negative serial blood/ urine cultures and no evidence for tuberculosis, HIV, CMV, toxoplasma, parvovirus, human herpesvirus 6 (HHV6), toxocara, or syphilis infection. EBV testing showed negative heterophile antibodies, but a positive (low) viral load by polymerase chain reaction (313 copies $/ \mathrm{mL}$ ) and capsid and nuclear antigen IgG; however, the lack of EBV-IgM suggested a remote infection. Hepatitis serologies were negative for hepatitis $C$ virus and hepatitis $B$ virus and positive for hepatitis $A$ virus $\lg G$. Serologies for rheumatoid factor (RF) and ANA were negative. Thyroid-stimulating hormone was within normal limits. Abdominal ultrasound revealed normal spleen and no hepatobiliary abnormalities.

On hospital admission day 5, the patient underwent excisional biopsy of her left posterior lymph node, and subsequent cytogenetics showed no evidence of lymphoma. Immunopathology revealed a distorted architecture with multifocal, paracortical areas of apoptotic necrosis with abundant karyorrhectic debris and numerous histiocytes of different types at the edge of the necrotic foci (Fig. 2A-C). These areas were devoid of neutrophils and plasma cells but had abundant $\mathrm{CD}^{+} 8^{+}$histiocytes (Fig. 2D-F). There was no evidence of malignancy, and special stains for fungi, mycobacteria, bacteria, and 

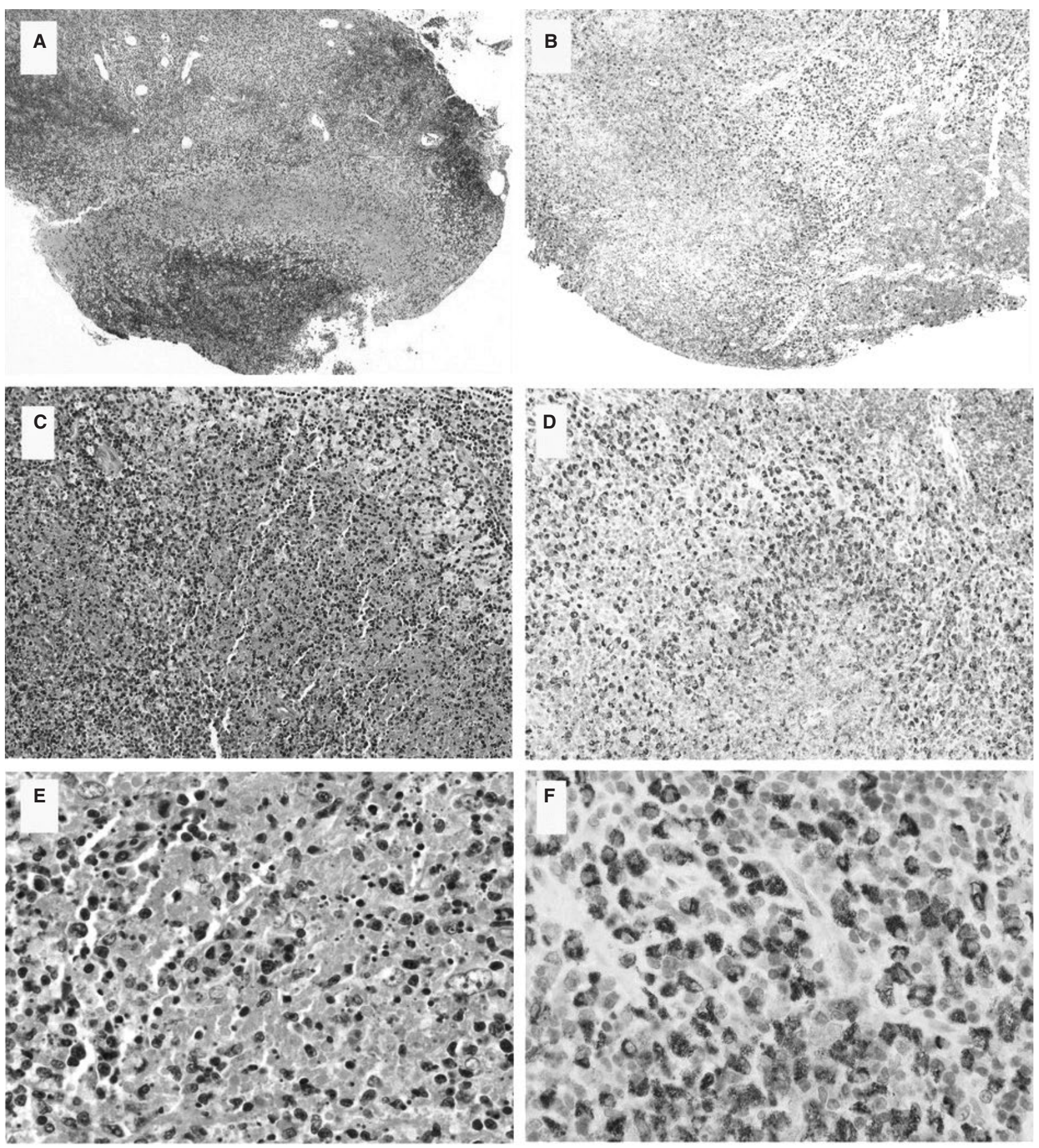

Figure 2. Histopathology of excised posterior cervical lymph node. Right column (A-C), H and E stain; left column (D-F), CD68 staining, for histiocytes and dendritic cells. Panel a shows affected lymph node architecture that is partially effaced by paracortical expansion composed of circumscribed foci of apoptotic necrosis with abundant karyorrhectic debris and numerous histiocytes of different types at the edge of the necrotic foci. Panels $b$ and $c$ demonstrate necrotizing lymphadenitis devoid of granulomas, neutrophils, bacteria or Epstein-Barr virus, and abundant eosinophilic fibrinoid and nuclear debris. Panels D-F demonstrate a prominent infiltration of CD68 ${ }^{+}$ histiocytes. Specific stains with $\alpha$ CD123 for plasmacytoid dendritic cells or $\alpha$ CD8 for T cells were not performed, although these cells are expected to be present in high numbers around the areas of necrosis. 
Reports on Kikuchi's Necrotizing Histiocytic Lymphadenitis

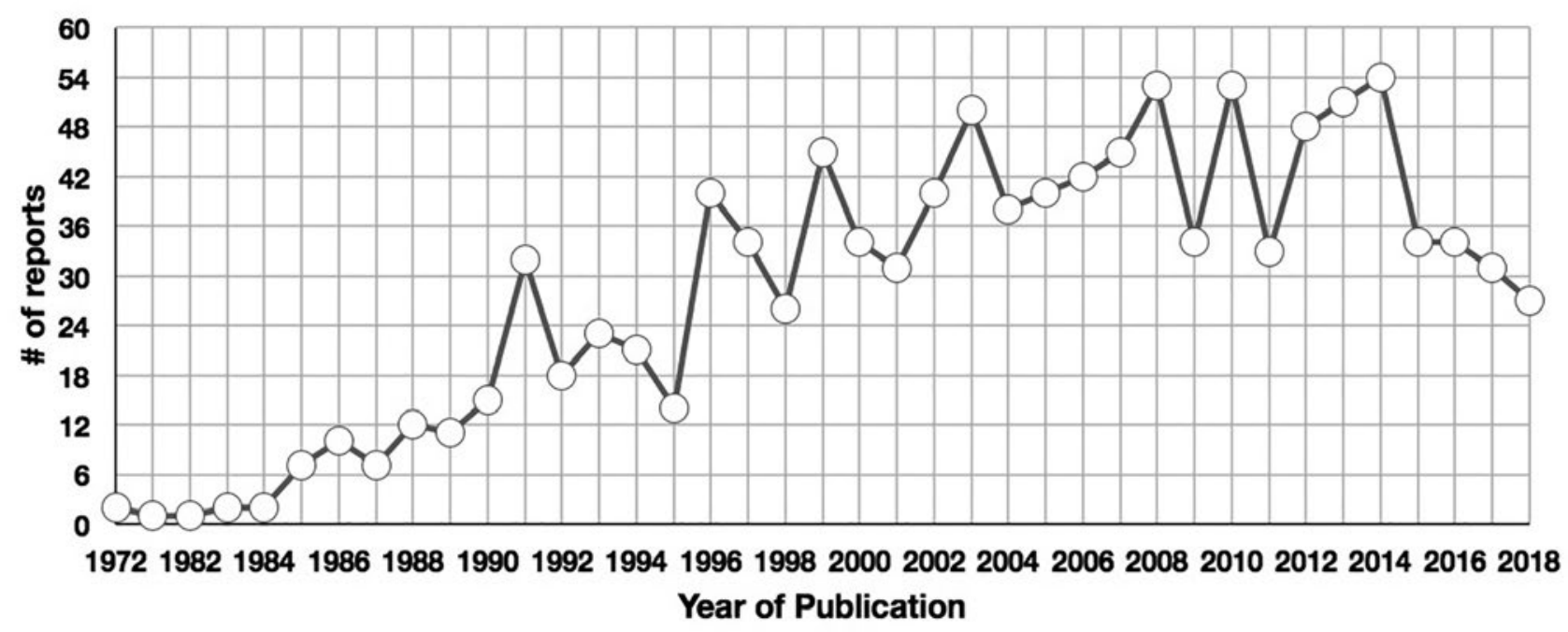

Figure 3. The Web of Science database was searched with the following criteria: ALL DATABASES; Topic=(histiocytic necrotizing lymphadenitis); or Topic=(Kikuchi's lymphadenitis); Refined by: General Categories=(SCIENCE TECHNOLOGY) and Publication Years=All Years. Total number of reports since the first publication in 1972 is 974 . IMBIOMED database search (keyword "Kikuchi") revealed 14 publications since 1997.

EBV were all negative. The pathology of benign histiocytic necrotizing lymphadenitis confirmed the diagnosis of KFD. Throughout her admission, the patient's AST and ALT remained mildly elevated; her anemia persisted as well as her protein gap, and she continued to have fatigue, tender $L A D$, and myalgias, which were managed with analgesics. Outpatient follow-up showed gradual improvement of $L A D$ and fevers, but the patient continued to have intermittent joint pains and fatigue 4 months later.

\section{DISCUSSION}

Kikuchi-Fujimoto histiocytic necrotizing lymphadenitis is a rare, benign, and usually self-limiting disease of unknown cause that commonly affects young Asian women $^{3}$. Most knowledge of this disease process comes from case reports, which indicate that there is a slight gender preference, a broad ethnic/racial distribution, and an age range of 6-80 years with most cases occurring under 40 years of $\mathrm{age}^{3}$. The incidence of KFD in the literature has markedly increased since its first description in 1982, with 27-34 reports annually from around the world in the past 3-4 years (Fig. 3). It is difficult to determine if this reflects a true rise in disease incidence or, alternatively, that clinicians are becoming increasingly more aware of this rare cause of FUO.

The most common symptoms of KFD are fever, cervical $L A D$, fatigue, and joint pain, all of which were present in this patient. Other findings are night sweats, nausea, vomiting, and diarrhea. The presentation, course, and histology of KFD suggest an immune response of CD8 T cells and histiocytes to an infectious agent, such as EBV, HHV6, HHV8, HIV, parvovirus, and others, with EBV being the most suspected ${ }^{4}$. Our patient showed evidence of a remote EBV infection consistent with this hypothesis. Laboratory findings in KFD are usually normal except for elevated ESR, neutropenia, anemia, and elevated $\mathrm{LDH}^{3}$, which presented in our patient. Although ANA and RF are usually negative, KFD has been proposed as a rare manifestation of systemic lupus erythematosus (SLE) lymphadenitis, as it co-occurs with SLE, and many patients with KFD go on to develop SLE ${ }^{5}$. Histopathology can differentiate KFD and SLE: SLE nodes show follicular hyperplasia with granulocytes and plasma cells that are not found in KFD. Supportive measures with analgesics and antipyretics are the standard of care for KFD. Symptoms usually resolve within 1-4 months; however, severe presentations have been shown to improve on steroids or IVIG. All patients should be followed for potential recurrence (rare) and/or development of SLE. 
The chief complaint of our patient was persistent fevers and cervical tender LAD. FUO with associated LAD usually skews the differential diagnosis toward infectious or malignant causes. Up to $56 \%$ of adult physical examinations can reveal palpable cervical lymph nodes, with the most common cause being infection ${ }^{6}$. Fluctuant cervical nodes are associated with bacterial infections, while those that are hard may reflect malignancy. Prolonged LAD is associated with atypical mycobacteria, cat-scratch disease, toxoplasmosis, KFD, sarcoidosis, and Kawasaki's syndrome, which can be confused with neoplasms 6 . If laboratory tests have not pointed toward a diagnosis, lymph node biopsy with histology can determine an etiology or at least rule out malignancy.

In the present case, the 11/2-month postponement of histopathological studies led to suboptimal care reflected by a delayed diagnosis, an extensive inpatient infectious work-up, unnecessary interventions, and hospitalization time. The critical question facing clinicians with a febrile patient with LAD that has an initial negative laboratory work-up is: When is biopsy indicated? Current recommendations suggest performing a biopsy when abnormal lymph nodes have not resolved after 4 weeks or when systemic signs (weight loss, night sweats, and fevers) suggest malignancy ${ }^{2}$. In the setting of FUO, Iymph node biopsy can establish a diagnosis in up to $80 \%$ of cases $^{7}$ making this procedure extremely relevant in the initial work-up of FUO with LAD. Models for the work-up of LAD have been proposed but not tested in prospective studies. A model for young people predicts the need for biopsy in up to $97 \%$ of cases based on abnormal chest X-ray, LAD >2 cm, and ear/nose/throat symptoms ${ }^{8}$. A similar model in adults predicted the need for biopsy if the patient presented with age $>40$ years, tenderness, larger size, generalized pruritus, supraclavicular location, and hard texture, with excellent sensitivity but poor specificity ${ }^{9}$

Once the decision for lymph node analysis has been made, the clinician must choose between less invasive fine-needle aspiration (FNA) or more invasive excisional biopsy. FNA is a quick, accurate, cost-effective, and safe diagnostic technique that can rapidly provide diagnostic clues and alleviate patient anxiety from a malignant diagnosis. The overall reported diagnostic accuracy for lymph node FNAs is approximately 90\%, with an $85-95 \%$ sensitivity and $98-100 \%$ specificity ${ }^{10}$. However, KFD diagnosis can be missed by FNA alone, with only $56 \%$ accuracy, $38 \%$ false-positives, and $50 \%$ false negatives ${ }^{10}$. Although the excisional biopsy is an invasive procedure, requiring local and/or general anesthesia, it has the greatest diagnostic yield and should be considered when FNA is unequivocal and has ruled out malignancy.

\section{CONCLUSIONS}

This case report exemplifies the diagnostic challenge for patients presenting with FUO and cervical $L A D$. KFD is a rare autoimmune disease affecting mostly young adults with the cardinal symptoms of fever and cervical LAD, and its diagnosis is based on a characteristic histiocytic necrotizing lymphadenitis identified by lymph node biopsy. Despite its ability to expedite diagnoses and rule out malignancy, lymph node biopsy remains an underutilized tool, often relegated to the end of extensive laboratory work-ups. As demonstrated in this report, postponing lymph node biopsy may delay diagnosis and optimal care. Given that posterior cervical LAD is more specific to EBV infection, tuberculosis, Iymphoma, KFD, or head and neck malignancies, we propose that clinical practice guidelines recommend a lymph node biopsy, by FNA or excision, be done early in the diagnostic work-up of patients with FUO and posterior cervical LAD.

\section{AUTHORS' CONTRIBUTIONS}

AGB and VHD were responsible for diagnosis, patient management, literature review, and writing. All authors read and approved the final manuscript.

\section{ACKNOWLEDGMENT}

We thank the patient for consenting to the publication of this case report.

\section{REFERENCES}

1. Bleeker-Rovers CP, Vos FJ, de Kleijn EM, et al. A prospective multicenter study on fever of unknown origin: the yield of a structured diagnostic protocol. Medicine (Baltimore). 2007;86:26-38. 
2. Habermann TM, Steensma DP. Lymphadenopathy. Mayo Clin Proc. 2000;75:723-32.

3. Hutchinson CB, Wang E. Kikuchi-Fujimoto disease. Arch Pathol Lab Med. 2010;134:289-93.

4. Hudnall SD, Chen T, Amr S, Young KH, Henry K. Detection of human herpesvirus DNA in Kikuchi-Fujimoto disease and reactive lymphoid hyperplasia. Int J Clin Exp Pathol. 2008:1:362-8.

5. Khanna D, Shrivastava A, Malur PR, Kangle R. Necrotizing lymphadenitis in systemic lupus erythematosus: is it Kikuchi-Fujimoto disease? J Clin Rheumatol. 2010;16:123-4.

6. Bazemore AW, Smucker DR. Lymphadenopathy and malignancy. Am Fam Physician. 2002;66:2103-10.
7. Bentz MH, Dupond JL. Diagnostic value of lymphadenopathy associated with fever and inflammation of unknown origin: a study of 69 patients. Rev Med Int. 2011;32:461-6.

8. Slap GB, Connor JL, Wigton RS, Schwartz JS. Validation of a mode to identify young patients for lymph node biopsy. JAMA. 1986;255: 2768-73.

9. Tokuda Y, Kishaba Y, Kato J, Nakazato N. Assessing the validity of a model to identify patients for lymph node biopsy. Medicine (Baltimore). 2003;82: 414-8.

10. Monaco SE, Khalbuss WE, Pantanowitz L. Benign non-infectious causes of lymphadenopathy: a review of cytomorphology and differential diagnosis. Diagn Cytopathol. 2012;40:925-38. 